\title{
COMMENT
}

\section{Repurposing azithromycin for neuroprotection in neonates}

\author{
Raul Chavez-Valdez ${ }^{1}$ \\ Pediatric Research (2019) 86:423-424; https://doi.org/10.1038/s41390-019-0443-3
}

In this issue of Pediatric Research, Barks and his colleagues from the University of Michigan tested the effectiveness of intraperitoneal (i.p.) azithromycin as an anti-inflammatory post-injury (rescue) neuroprotectant in the P7 (near-term equivalent) and P10 (term equivalent) rat model of neonatal hypoxia-ischemia $(\mathrm{HI})$ brain injury using the Vannucci model. Based on their experimental results, Barks et al. concluded that a 3-dose regimen of azithromycin delayed to $2 \mathrm{~h}$ after the $\mathrm{HI}$ insult and continued for 2 more doses (24 hours apart) was superior than control (normal saline) or single dose. Azithromycin affords protection against: (i) sensorimotor deficits and (ii) cortical, striatal, and hippocampal injury (P7 model) or cortical injury (P10 model) at P35. The efficacy of azithromycin was dose dependent and the neuroprotection was lost if therapy was delayed further than $2 \mathrm{~h}$ after the $\mathrm{HI}$ insult. ${ }^{1}$ This is a well-designed and extremely timely paper, discussing once again the effectiveness of repurposed medications in an attempt to provide neuroprotection following perinatal brain injury. The strategy of repurposing available medications for new indications speeds the translation from bench to bedside. ${ }^{2,3}$ One of the putative mechanisms of action of azithromycin likely includes the drug-induced polarization of macrophages toward M2 phenotype, limiting blood-brain barrier damage and resulting in decreased infiltration of neurotrophils and mononuclear cells to the brain, as speculated from the stroke model of transient middle cerebral artery occlusion and i.p. azithromycin treatment by Amantea et al. ${ }^{4}$ Neuroprotection linked to the treatment with azithromycin is also reported in models of retinal ischemic injury in rats. ${ }^{5}$ Thus, azithromycin (and other macrolides) appears to provide neuroprotection against ischemic, hypoxic-ischemic, and ischemic-reperfusion brain injuries in models of preconditioning and post-injury treatment as shown by Barks et al. and others. ${ }^{1,4,6,7}$

The molecular pathway targeted by azithromycin to provide neuroprotection to the developing brain remains elusive and it is not the focus of this paper by Barks and colleagues. However, azithromycin appears to limit the translocation of nuclear factor (NF)-KB and activator protein (AP)-1 to the nucleus, known transcription factors for pro-inflammatory cytokine expression, resulting in their downregulation in glial cells in a poly [ADP-ribose] polymerase (PARP)-1-independent manner. ${ }^{8}$ Since the PARP-1mediated molecular pathway preferentially produces necrotic-like cell death after neonatal $\mathrm{HI}$ injury in males mice, ${ }^{9-11}$ the lack of PARP-1 dependence in the mechanism of action of azithromycin could explain the lack of sexual dimorphism reported by Barks et al. ${ }^{1}$ Another mechanistic aspect not addressed is the effects of the known cardioprotection provided by azithromycin in models of myocardial infarction. ${ }^{12}$ The effect of the improved cerebral perfusion resulting from improved cardiac output may account for some of the neuroprotection reported by Barks et al. ${ }^{1}$.
Despite the very compelling and promising data presented by Barks and colleagues, we can argue against repurposing antibiotics, such as azithromycin without appropriate extended safety data. Among many aspects that makes the use of broadspectrum antibiotics for indications other than treatment of infections problematic is that their broad use can lead to the emergence of microbial resistance, dysbiosis, and their consequences. ${ }^{13-21}$ To limit the emergence of resistance, Zhang et al. ${ }^{22}$ have proposed to biochemically modify azithromycin molecule to maintain its ability to modulate macrophage phenotype toward a less pro-inflammatory cytokine-releasing state but limiting the binding of the molecule to the bacterial ribosome and thus decreasing the risk for development of antibiotic resistance. The neuroprotective properties of these modified compounds derived from azithromycin will need further investigation in models of neonatal HI. The second unintended consequence of repurposing broad-spectrum antibiotics, the development of dysbiosis in the neonate, has very serious life-long health consequences. ${ }^{23-25}$ The use of azithromycin pre-natally for prophylaxis during premature labor or prior to cesarean section may decrease the risk of maternal chorioamnionitis ${ }^{26}$; however, the consequences in the fetus and the newborn are still grossly unknown. Now more than ever, thanks to antibiotic stewardship programs in most hospitals, the focus is on appropriate use of antibiotics to reduce microbial resistance and limit other better-understood side effects. Thus using antibiotics for indications other than treatment of confirmed infections will require compelling basic, translational and clinical data to limit the risk in the population targeted for its use.

Although azithromycin has good tolerance and safety profile in children aged $>2$ years, ${ }^{27-29}$ it is important to recognize that azithromycin neither has been properly studied for safety in neonates, nor has been approved for children aged $<6$ months, with the only exception being to treat pertussis. ${ }^{30}$ Furthermore, not unlike many other drugs commonly used in neonates, there is even less data about their use in patients suffering of perinatal $\mathrm{HI}$ injury with multiorgan failure treated with therapeutic hypothermia. To date, most of the safety data about azithromycin derive from observational studies and randomized clinical trials to using the drug to decrease the risk of bronchopulmonary dysplasia in premature infants. ${ }^{31-33}$ Recently, the safety of azithromycin in that specific group of extreme low birth weight neonates was analyzed in a systematic review by Smith el al. ${ }^{34}$ Common adverse events associated with azithromycin were mostly gastrointestinal, including hypertrophic pyloric stenosis, which occurs in $2-3 \%$ of all treated patients. On the other hand, arrhythmias, commonly reported with the use of other macrolides, such as erythromycin, were only reported with azithromycin overdose. ${ }^{35}$ Azithromycin's half-life is estimated to be between 26 and $83 \mathrm{~h}$ in healthy neonates, ${ }^{36}$ but a dose and regimen has not

${ }^{1}$ Division of Neonatal-Perinatal Medicine, Department of Pediatrics, Johns Hopkins School of Medicine, Baltimore, MD, USA

Correspondence: Raul Chavez-Valdez (rchavez2@jhmi.edu)

Received: 9 May 2019 Accepted: 16 May 2019

Published online: 26 May 2019 
been established in patients suffering from perinatal $\mathrm{HI}$ injury with impaired liver and renal function and who are being treated with therapeutic hypothermia. Thus the compelling animal data reported by Barks and colleagues showing no increased mortality, temperature instability, or impaired weight gain in rodents receiving delayed azithromycin treatment, which provides functional and histopathological protection, provide enough evidence to make plausible to study azithromycin in a pre-clinical model of neonatal $\mathrm{HI}$ and therapeutic hypothermia. The ultimate question is whether delayed azithromycin treatment supplements the effect of therapeutic hypothermia alone. If so, extensive data about the safety of azithromycin in this vulnerable population would be necessary before considering its clinical use for neuroprotection.

\section{ADDITIONAL INFORMATION}

Competing interests: The author declares no competing interests.

Statement of ethics: We confirm that any aspect of the work covered in this manuscript involving experimental animals has been conducted with the ethical approval of all relevant bodies.

Publisher's note: Springer Nature remains neutral with regard to jurisdictional claims in published maps and institutional affiliations.

\section{REFERENCES}

1. Barks J.D.E, Liu Y., Wang L., Pai M.P., Silverstein F.S. Repurposing azithromycin for neonatal neuroprotection. Pediatr. Res. (2019). https://doi.org/10.1038/s41390019-0408-6 [Epub ahead of print].

2. McCoy, T. H. Jr. \& Perlis, R. H. A tool to utilize adverse effect profiles to identify brain-active medications for repurposing. Int. J. Neuropsychopharmacol. 18, pyu078 (2015).

3. Amantea, D. \& Bagetta, G. Drug repurposing for immune modulation in acute ischemic stroke. Curr. Opin. Pharmacol. 26, 124-130 (2016).

4. Amantea, D. et al. Azithromycin protects mice against ischemic stroke injury by promoting macrophage transition towards M2 phenotype. Exp. Neurol. 275(Pt 1), 116-125 (2016).

5. Varano, G. P. et al. Post-ischemic treatment with azithromycin protects ganglion cells against retinal ischemia/reperfusion injury in the rat. Mol. Vis. 23, 911-921 (2017).

6. Inaba, T., Katayama, Y., Ueda, M. \& Nito, C. Neuroprotective effects of pretreatment with macrolide antibiotics on cerebral ischemia reperfusion injury. Neurol. Res. 37, 514-524 (2015).

7. Amantea, D., Certo, M., Petrelli, F. \& Bagetta, G. Neuroprotective properties of a macrolide antibiotic in a mouse model of middle cerebral artery occlusion: characterization of the immunomodulatory effects and validation of the efficacy of intravenous administration. Assay Drug Dev. Technol. 14, 298-307 (2016).

8. Petrelli, F., Muzzi, M., Chiarugi, A., Bagetta, G. \& Amantea, D. Poly(ADP-ribose) polymerase is not involved in the neuroprotection exerted by azithromycin against ischemic stroke in mice. Eur. J. Pharmacol. 791, 518-522 (2016).

9. Hagberg, $H$. et al. PARP-1 gene disruption in mice preferentially protects males from perinatal brain injury. J. Neurochem. 90, 1068-1075 (2004).

10. Northington, F. J. et al. Necrostatin decreases oxidative damage, inflammation, and injury after neonatal HI. J. Cereb. Blood Flow Metab. 31, 178-189 (2011).

11. Chavez-Valdez, R., Martin, L. J., Razdan, S., Gauda, E. B. \& Northington, F. J. Sexual dimorphism in BDNF signaling after neonatal hypoxia-ischemia and treatment with necrostatin-1. Neuroscience 260, 106-119 (2014).
12. Al-Darraji, A. et al. Azithromycin therapy reduces cardiac inflammation and mitigates adverse cardiac remodeling after myocardial infarction: potential therapeutic targets in ischemic heart disease. PLOS ONE 13, e0200474 (2018).

13. Parker, E. P. K. et al. Changes in the intestinal microbiota following the administration of azithromycin in a randomised placebo-controlled trial among infants in south India. Sci. Rep. 7, 9168 (2017).

14. Azad, M. B., Bridgman, S. L., Becker, A. B. \& Kozyrskyj, A. L. Infant antibiotic exposure and the development of childhood overweight and central adiposity. Int J. Obes. (Lond.) 38, 1290-1298 (2014).

15. Arrieta, M. C. et al. Early infancy microbial and metabolic alterations affect risk of childhood asthma. Sci. Transl. Med. 7, 307ra152 (2015).

16. lizumi, T. et al. Effect of antibiotic pre-treatment and pathogen challenge on the intestinal microbiota in mice. Gut Pathog. 8, 60 (2016).

17. Blaser, M. J. \& Dominguez-Bello, M. G. The human microbiome before birth. Cell Host Microbe 20, 558-560 (2016).

18. Livanos, A. E. et al. Antibiotic-mediated gut microbiome perturbation accelerates development of type 1 diabetes in mice. Nat. Microbiol. 1, 16140 (2016).

19. Blaser, M. J. Antibiotic use and its consequences for the normal microbiome. Science 352, 544-545 (2016).

20. Mahana, D. et al. Antibiotic perturbation of the murine gut microbiome enhances the adiposity, insulin resistance, and liver disease associated with high-fat diet. Genome Med. 8, 48 (2016).

21. O'Brien, K. S. et al. Antimicrobial resistance following mass azithromycin distribution for trachoma: a systematic review. Lancet Infect. Dis. 19, e14-e25 (2019).

22. Zhang, B. et al. Macrolide derivatives reduce proinflammatory macrophage activation and macrophage-mediated neurotoxicity. CNS Neurosci. Ther. 25, 591-600 (2019).

23. Willing, B. P., Russell, S. L. \& Finlay, B. B. Shifting the balance: antibiotic effects on host-microbiota mutualism. Nat. Rev. Microbiol. 9, 233-243 (2011).

24. Bedford Russell, A. R. \& Murch, S. H. Could peripartum antibiotics have delayed health consequences for the infant? BJOG 113, 758-765 (2006).

25. Warner, B. B. et al. Gut bacteria dysbiosis and necrotising enterocolitis in very low birthweight infants: a prospective case-control study. Lancet 387, 1928-1936 (2016).

26. Tita, A. T. et al. Adjunctive azithromycin prophylaxis for cesarean delivery. New Engl. J. Med. 375, 1231-1241 (2016).

27. Hopkins S Clinical toleration and safety of azithromycin. Am. J. Med. 91, 40S-45S (1991).

28. Langtry, H. D. \& Balfour, J. A. Azithromycin. A review of its use in paediatric infectious diseases. Drugs 56, 273-297 (1998).

29. Ruuskanen, O. Safety and tolerability of azithromycin in pediatric infectious diseases: 2003 update. Pediatr. Infect. Dis. J. 23, S135-S139 (2004).

30. Tiwari, T., Murphy, T. V., Moran, J. \& National Immunization Program CDC. Recommended antimicrobial agents for the treatment and postexposure prophylaxis of pertussis: 2005 CDC Guidelines. MMWR Recomm. Rep. 54, 1-16 (2005).

31. Ballard, H. O. et al. Use of azithromycin for the prevention of bronchopulmonary dysplasia in preterm infants: a randomized, double-blind, placebo controlled trial. Pediatr. Pulmonol. 46, 111-118 (2011).

32. Ballard, H. O., Bernard, P., Qualls, J., Everson, W. \& Shook, L. A. Azithromycin protects against hyperoxic lung injury in neonatal rats. J. Invest. Med. 55, 299-305 (2007).

33. Viscardi, R. M. et al. Azithromycin to prevent bronchopulmonary dysplasia in ureaplasma-infected preterm infants: pharmacokinetics, safety, microbial response, and clinical outcomes with a 20-milligram-per-kilogram single intravenous dose. Antimicrob. Agents Chemother. 57, 2127-2133 (2013)

34. Smith, C. et al. Use and safety of azithromycin in neonates: a systematic review. BMJ Open 5, e008194 (2015)

35. Tilelli, J. A., Smith, K. M. \& Pettignano, R. Life-threatening bradyarrhythmia after massive azithromycin overdose. Pharmacotherapy 26, 147-150 (2006).

36. Hammerschlag, M. R., Gelling, M., Roblin, P. M., Kutlin, A. \& Jule, J. E. Treatment of neonatal chlamydial conjunctivitis with azithromycin. Pediatr. Infect. Dis. J. 17, 1049-1050 (1998). 